1 ECOLOGICAL INFORMATICS (ISSN: 1574-9541) 32: pp. 63-68. (2016)

2

\title{
Once again on the components of pairwise beta diversity
}

4

$5 \quad$ Running title: On components of pairwise beta diversity

6 Word count: ca. 5400

7

8 Authors:

9 János Podani

10 Department of Plant Systematics, Ecology and Theoretical Biology, Institute of

11 Biology, L. Eötvös University, Pázmány P. s. 1/c, H-1117, Budapest, Hungary

12 and

13 Ecology Research Group of the Hungarian Academy of Sciences, Budapest, Hungary

14 Corresponding author. Email: podani@ludens.elte.hu

15

16 and

17

18

Dénes Schmera

19 MTA Centre for Ecological Research, Tihany, Hungary

20 and

21 Section of Conservation Biology, Department of Environmental Sciences, University

22 of Basel, Basel, Switzerland

23

24 
Presence-absence based beta diversity defined for pairs of sites may be partitioned into components following two different ways of thinking. Within the framework of Baselga (abbreviated hereafter as BAS), nestedness is crucial and dissimilarity is partitioned into replacement (turnover) and nestedness-resultant fractions. The method proposed by Podani and Schmera (POD), however, places emphasis on the mathematical additivity of components and divides dissimilarity into replacement and richness difference components. A recent comparison by Baselga and Leprieur (2015), on the example of the Jaccard family of indices, emphasizes the independence of replacement component from absolute richness difference and concludes that the replacement function of the BAS framework is the only true measure of species replacement. As a response to this study, we show here that 1) the sacrifice one must make for independence is that the components themselves are scaled differently and are not always comparable ecologically, 2) absolute (raw) replacement and richness difference are not independent, so that independence from the latter cannot be a fundamental criterion that a replacement measure should satisfy, 3) relativization applied in the POD framework is ecologically interpretable, leading to a meaningful conceptualization of species replacement, 4) the BAS and POD methods are linked through a generalized replacement function, 5) both the BAS and POD approaches may produce high correlations with environmental variables, whereas 6) the POD approach offers in many respects more illuminating demonstrations of the underlying changes of pattern than the graphs of Baselga and Leprieur for both artificial and actual fish distribution data.

48 Keywords: Data structure; Diversity partitioning; Nestedness; Richness difference;

49 Species replacement; Turnover 


\section{Introduction}

51 Describing species distribution patterns and underlying mechanisms is at the heart of

52 current biodiversity research. Beta diversity, summarising a wide variety of

53 conceptual and methodological approaches, is a key term for assessing community

54 variation in space and time. Baselga (2010) suggested that beta diversity can be

55 partitioned into spatial turnover (also termed replacement) and nestedness resultant

56 dissimilarity components. This partitioning framework (hereafter abbreviated as BAS,

57 following Legendre 2014) has been considered as a major contribution to this field

58 because the components may reflect the existence of different underlying mechanisms

59 driving beta diversity and thus the methodological framework can efficiently be used

60 for understanding central geographic, ecological and conservation issues.

61 Thanks to its novelty and soundness, this scheme triggered critical comments

62 (Schmera \& Podani 2011, Almeida-Neto et al. 2012), an intensive dispute (Baselga

63 2012, Carvalho et al. 2013, Chen 2015) and the development of competing

64 approaches (Podani \& Schmera 2011, Carvalho et al. 2012, Cardoso et al. 2014) as

65 well. For instance, according to a recent proposal, beta diversity can be partitioned

66 into replacement and richness difference components (hereafter abbreviated as POD,

67 following Legendre 2014, see also Podani \& Schmera 2011, Carvalho et al. 2012,

68 Carvalho et al. 2013). A review by Legendre (2014) compared these frameworks and

69 found that they represent alternative approaches with different conceptual and

70 mathematical backgrounds.

71 Most recently, Baselga \& Leprieur (2015) revisited the issue by evaluating the

72 performance of the BAS and POD frameworks under different conditions. They

73 emphasized that (1) the replacement components of the two approaches formalize

74 different concepts, and (2) only the replacement component of the BAS framework is 
independent of richness difference. Based on their simulations and analyses of actual data, BAS proved to be the winner, leading to the conclusion that (3) only its replacement component is able to express "true" replacement. We disagree with the final conclusion by questioning the basic assumption that the replacement component should be independent of richness difference and by arguing that the relevant component of the POD framework expresses replacement at least as faithfully as the BAS framework. Regarding independence, we refer to Chao et al. (2012) who clarified the multiple meaning of this term. Here, we mean that knowing one diversity component one has no information about the value of another, and vice versa. Our disagreement is also due to the fact that in many instances Baselga \& Leprieur (2015) still misunderstood, confused or neglected some basic aspects of the POD approach. To avoid the undesirable situation that potential users of either method are misguided, this rebuttal attempts to clarify the most important points.

\section{The BAS and POD frameworks}

For the benefit of the reader, we give a brief summary of the two frameworks

following the Legendre's (2014) system of symbols. Decomposition of beta diversity

91 for a pair of sites, expressed by the Sorensen dissimilarity index $\left(D_{S}\right)$ was first suggested by Baselga (2010):

93

$$
\mathrm{D}_{\mathrm{S}}=\frac{b+c}{2 a+b+c}=\operatorname{Repl}_{\mathrm{BS}}+\operatorname{Nes}_{\mathrm{BS}}=\frac{\min (b, c)}{a+\min (b, c)}+\frac{a}{a+\min (b, c)} * \frac{|b-c|}{2 a+b+c}
$$
in which $a$ refers to the number of species present in both sites, while $b$ and $c$ correspond to the number of species present only in the first and the second, respectively, such that $n=a+b+c$. The above equation implies that Simpson

99 dissimilarity $\left(\right.$ Repl $\left._{\mathrm{BS}}\right)$, expressing spatial turnover is subtracted from Sorensen 
100

101

102

103

104

105

106

107

$\beta_{\mathrm{WB}}=b+c=$ replacement + richness difference $=2 \min (b, c)+|b-c|$

$$
\mathrm{D}_{\mathrm{J}}=\frac{b+c}{a+b+c}=\operatorname{Repl}_{\mathrm{BJ}}+\mathrm{NeS}_{\mathrm{BJ}}=\frac{2 \min (b, c)}{a+2 \min (b, c)}+\frac{a}{a+2 \min (b, c)} * \frac{|b-c|}{a+b+c}
$$

The POD framework consists of an absolute (or raw) and a relativized decomposition of beta diversity (Podani and Schmera 2011). In the absolute decomposition, Weiher Boylen beta diversity ( $\beta_{\mathrm{WB}}$, see also Koleff et al 2003) is divided into (raw) replacement and (raw) richness difference components:

114 The basis of the relativized POD framework (Podani and Schmera 2011) is the

115 decomposition of pairwise beta expressed by the Jaccard dissimilarity $\left(\mathrm{D}_{\mathrm{J}}\right)$ into two

116 additive fractions according to

$$
\mathrm{D}_{\mathrm{J}}=\frac{b+c}{a+b+c}=\operatorname{Repl}_{\mathrm{J}}+\operatorname{Rich}_{\mathrm{J}}=\frac{2 \min (b, c)}{a+b+c}+\frac{|b-c|}{a+b+c} .
$$

The first part was termed the relativized species replacement $\left(\operatorname{Repl}_{\mathrm{J}}\right)$, while the second

121 was relativized richness difference $\left(\operatorname{Rich}_{\mathrm{J}}\right)$. It is important to point out that we never 122 considered these components as stand-alone dissimilarity functions, only fractions of 123 dissimilarity! According to our proposal, these two fractions, together with the 
124 complement of $D_{J}$, i.e., Jaccard similarity $\left(S_{J}\right)$ may be used as scores to specify the 125 position of the site pair in a 2D simplex diagram. If the same operation is done for all 126 pairs of sites in a region, then we have a tool for visualizing structure of the entire 127 presence/absence matrix (Podani and Schmera 2011). Sorensen index may also be 128 decomposed in similar manner (Carvalho et al. 2012, Legendre 2014), which is not 129 detailed here because 1) the only difference from Eq. 3 is that $2 a$ replaces $a$ in the 130 denominators and 2) this paper is concerned only with the Jaccard family of indices,

131 in response to Baselga \& Leprieur (2015) whose study is restricted to this group.

\section{The relativized replacement component in POD}

133 Before we discuss the relative merits and disadvantages of the two frameworks, a

134 terminological clarification is in order. This is important because Baselga and 135 Leprieur erroneously equate our relativized species replacement function $\left(\mathrm{Repl}_{\mathrm{J}}\right.$, in 136 Eq. 3) with Williams' replacement index (see $\beta_{-3}$ in their Table 1). The formula 137 proposed by Williams (1996) reads:

$\beta_{-3}=1-\frac{a+\min (b, c)+|b-c|}{a+b+c}=\frac{\min (b, c)}{a+b+c}$,

141 that is, $2 \beta_{-3}=R e p l_{\mathrm{J}}$, a fact that cannot be neglected when our objective is

142 decomposition into additive fractions. Multiplication by 2 was first suggested by

143 Cardoso et al. (2009) to ensure that the index changes between 0 and 1 . Zero obtains

144 in the situation when there are no unique species for either site or both so that there is

145 no replacement. The maximum value results if the species set of one site is

146 completely replaced by the species of the other, which is possible only if $b=c>0$ and $147 \quad a=0$. 
148 Cardoso et al. (2009) used the same abbreviation $\left(\beta_{-3}\right)$ for the multiplied index,

149 whereas Podani \& Schmera (2011) suggested $\frac{2 \min (\mathrm{b}, \mathrm{c})}{\mathrm{a}+\mathrm{b}+\mathrm{c}}$ as a relativized replacement

150 index without referring to the suggestions of Cardoso et al. (2009). Thus, it is partly

151 our fault that the multiplied version and the original Williams index are still confused.

152 However, since multiplication modifies the range of the measure, $\beta_{-3}$ and Repl $l_{\mathrm{J}}$ should

153 be treated as different functions. This is in agreement with Koleff et al. (2003) who

154 list several examples of beta diversity measures which differ only in a multiplying

155 factor of 2.

156 3. Commensurability

157 Decomposition of absolute or raw beta diversity has been rarely used in practice, 158 because the results from different surveys are hardly commensurable. Relativization 159 (or standardization) of raw values is a general strategy, as with dissimilarity 160 coefficients whose values range between 0 and 1 . When dissimilarities themselves are 161 partitioned, however, another aspect of commensurability enters the scene. If we 162 examine the suggested components of $D_{\mathrm{J}}$ in Eq. 2 we find that they disagree in the 163 manner they are standardized, as demonstrated by the following example with $a=3$, $164 b=5$ and $c=1 . \mathrm{D}_{\mathrm{J}}(=6 / 9)$ is the ratio of the number of unique species and the total 165 number of species in the two sites being compared, and $\operatorname{Repl}_{\mathrm{BJ}}(=2 / 5)$ may be 166 conceived as the ratio of the number of replaced species and the total number of 167 species if both sites were equally poor (i.e. with $b=c=1$ ). The third one, $\mathrm{Nes}_{\mathrm{BJ}}$ $168(=12 / 45)$ lacks any ecologically meaningful interpretation in terms of species numbers 169 or raw data values. Apparently, due to different relativization and weighting 170 operations applied to the component terms, there is no "common currency" for the 171 three terms involved. This is not so with the POD framework: $\mathrm{D}_{\mathrm{J}}$ and both of its 172 fractions in Eq. 3 are interpretable as ratios, since Repl $_{J}=2 / 9$ and Rich $_{J}=4 / 9$. These 
are expressed on the same scale as $\mathrm{D}_{\mathrm{J}}$ itself. We think this is important even though

174 the dissimilarities themselves are dimensionless quantities. A conclusion is that

175 whereas the BAS approach is inconsistent, the POD framework is consistent in the

176 way component terms are measured. This holds true for the partitioning of Sorensen

177 index as well (see Schmera \& Podani 2011). A natural question arises: what are then

178 the advantages of using inconsistent relativization in the BAS decomposition? We

179 look for the answer in the next section.

\section{Replacement and its independence from richness difference}

181 Baselga and Leprieur (2015) emphasize the main result of their analyses as follows:

182 the replacement component in the BAS framework is independent of richness

183 difference, whereas the analogous component in the POD approach is not. To prove

184 this, they apply a huge arsenal of statistical tools (comparison of parallel components,

185 random parameter method, the evaluation of joint probabilities etc., see their Figure

186 2). The above conclusion is straightforward and no detailed tests are required,

187 however, if we consider how Repl $l_{\mathrm{BJ}}$ (and $\mathrm{Repl} \mathrm{BS}_{\mathrm{BS}}$ ) are formulated. The denominator of

$188 \operatorname{Repl}_{\mathrm{BJ}}, a+2 \min (b, c)$, contains information on the number of species in the poorer site

189 whereas the number of species in the richer site $(a+\max (b, c))$ does not appear in the

190 formula at all. Therefore, the result is, by definition, unaffected by the richer site and

191 in turn richness difference either (see also Appendix 1). Obviously, Simpson

192 dissimilarity $\left(\mathrm{Repl}_{\mathrm{BS}}\right)$ and Baselga's Repl $\mathrm{BJ}_{\mathrm{BJ}}$ were constructed on purpose to satisfy

193 this external requirement. Richness difference as such is not part of their

194 decomposition procedure.

195 Baselga and Leprieur (2015) argued that the use of the term replacement for the two

196 respective components originating from different frameworks might be misleading

197 and suggested that the term replacement should be reserved to indices that are 
independent of richness difference. While we agree that terminological clarity is essential, we disagree with the suggestion itself. Here we show that independence from absolute richness difference cannot be a criterion of a replacement measure. In

201 fact, replacement "implies the replacement of some species by others" (p. 135,

202 Baselga 2010) and we emphasized several times (see e.g., p. 155 in Schmera and 203 Podani 2011) that species replacement originally refers to an absolute measure, i.e., to 204 the number of species that are replaced between two sites $(2 \min (b, c))$. This is not 205 independent mathematically from raw richness difference $(|b-c|)$ as proved in 206 Appendix 1 and graphically demonstrated also by two simulations in the 207 Supplementary Material using random parameter approaches similar to those in 208 Baselga and Leprieur's randomization experiments with relativized measures. For 209 actual ecological data, these two components may indeed be uncorrelated, yet large 210 richness differences rarely associate with large replacement due to the constraining 211 effect of the maximum number of species (see Supplementary Material). Therefore, if 212 the above suggestion were accepted, then even raw replacement could not be called 213 "replacement" - which is conflicting with any intuitive views on species turnover. A 214 potential source of this contradiction is that decomposition of absolute (raw) beta 215 diversity is not included originally in the BAS framework. In sum, independence 216 cannot be a fundamental criterion that a replacement measure should satisfy and thus

217 the replacement component of the BAS framework cannot be regarded as the sole 218 candidate for the use of the term replacement.

219 5. How to relativize?

220 Coefficients Repl $l_{\mathrm{BS}}$ and $\operatorname{Repl}_{\mathrm{BJ}}$ are asymmetric in handling $b$ and $c$, as shown by the 221 following example with $a=1, b=49$ and $c=1$. Now, Repl ${ }_{\mathrm{BJ}}=2 / 3$ suggesting high 222 level of turnover, which is fine with respect to the poorer site. It has only two species, 
223 and the loss of one species and the gain of another is indeed a significant change. As

224 Carvalho et al. (2013) pointed out, however, "we do not see any reason why only the

225 poorer site should establish the theoretical upper limit of species replacement." Thus,

226 from the "viewpoint" of the richer site, the same change is practically nothing if we

227 consider that it has as much as 50 species in total. One alternative is therefore a new

228 replacement function in the Jaccard index-family, which considers the richer site for

229 relativization (Eq. A1, in Appendix 2). This function takes the value of $0.02-$ which

230 appears a more reasonable score than $2 / 3$.

231 In these functions, one of the sites plays little or no role in determining the index

232 value - an undesirable property for a dissimilarity function. The relativized

233 replacement index Repl $_{\mathrm{J}}$ does provide a balanced result with respect to the total

234 species richness of the two sites. For the small example above, the value of $2 / 51 \sim$

2350.04 indicates fairly well that only a negligible minority of potential species

236 participated in the replacement process.

237 Relativization by $a+b+c$ has the obvious consequence that Repl will not be

238 independent of richness difference (Appendix 1). It is implicit in the denominator

239 (i.e., $n=a+2 \min (b, c)+|b-c|)$ therefore, if $n$ increases because $|b-c|$ increases then

240 the value of the index will decrease. If $|b-c|$ increases such that $n$ remains constant,

241 then $\min (b, c)$ must decrease after $a$ reached zero (and may decrease even before), so

242 that the value of the index also decreases. In the POD approach this is fundamental: $\mathrm{S}_{\mathrm{J}}$

243 and the two components of $\mathrm{D}_{\mathrm{J}}$ mutually constrain one another (their sum is always 1 ),

244 these three are expressed on the same scale to provide a meaningful 2D display in

245 form of a simplex plot, and the inclusion of all site pairs in the same simplex plot

246 would be impossible without relativization. Notably, such kind of relativization is

247 commonplace in numerical ecology - often leading to loss of independence. For 
example, if raw data are standardized by species totals, the scores, which were originally independent, will now be dependent: each new value will be affected by all

250 the other scores for the given species. Appendix 2 shows that the two asymmetric

251 replacement functions represent extreme cases of a general replacement formula in

252 which Repl $l_{\mathrm{J}}$ takes an intermediate position.

\section{6. Correlations with environmental variables}

254 Although we disagree with the view implicit in Baselga and Leprieur (2015)

255 suggesting that the higher the correlation between an index value and an

256 environmental variable, the better the index, we planned to repeat their study of actual

257 data (North American fish dataset) with more measures involved, including raw

258 replacement and richness difference. This would clarify the question whether it is

259 worth switching from raw measures to relativized ones. However, the supplementary

260 material did not include the geographical distances in terms of the number of drainage

261 basin divides between river basins Baselga and Leprieur (2015) were using.

262 Furthermore, glacial history was presented as an ordinal variable to which, according

263 to our view (Podani 2005), correlation measures cannot be applied (but see Ricotta

264 and Feoli 2013, for alternative views on the usefulness of ordinal data). Thus, we

265 computed correlations between the matrices of eight components in the BAS and

266 POD approaches and only four environmental variables, two of them being PCA

267 summaries of 17 climatic variables (Table 1).

269 Table 1. Pearson product moment matrix correlations between various coefficients

270 and components of beta diversity and four variables reflecting inter-site distances in

271 terms of climate (PCA1 and PCA2) plus altitude and area for the North American fish

272 distribution data. 


\begin{tabular}{lcccc}
\hline Measure & PCA1 & PCA2 & Altitude & \multicolumn{1}{l}{ Area } \\
\hline$|b-c|$ & 0.155 & -0.039 & -0.042 & 0.475 \\
$2 \min (b, c)$ & -0.082 & -0.041 & 0.038 & 0.138 \\
$\mathrm{D}_{\mathrm{J}}$ & 0.372 & 0.258 & 0.191 & 0.018 \\
$\mathrm{Rich}_{\mathrm{J}}$ & 0.246 & 0.020 & -0.018 & 0.102 \\
$\operatorname{Repl}_{\mathrm{J}}$ & -0.061 & 0.119 & 0.123 & -0.099 \\
$\operatorname{Repl}_{\mathrm{BJ}}$ & 0.367 & 0.284 & 0.216 & -0.126 \\
Nes $_{\mathrm{BJ}}$ & -0.248 & -0.213 & -0.167 & 0.191 \\
New function (A1) & 0.344 & 0.235 & 0.172 & 0.293 \\
\hline
\end{tabular}

273

274 It is seen from the table that the BAS framework does not have the privilege to

275 produce relatively high correlations. The largest value $(0.475)$ was obtained between

276 absolute richness difference and area difference - which agrees well with basic

277 biogeographical "rules". The effect of area was less conspicuous for other

278 components, with the new turnover function (Eq. A1) as an exception $(r=0.293)$.

279 Regarding the BAS and POD frameworks, Baselga's replacement function produced

280 higher correlations with climate than POD. For example, with the first climatic

281 component both the absolute and relativized turnover $\left(\operatorname{Repl}_{\mathrm{J}}\right)$ were slightly negatively

282 correlated, while Repl $l_{\mathrm{BJ}}$ and function $\mathrm{A} 1$, as well as $\mathrm{D}_{\mathrm{J}}$ itself had positive correlations

283 larger than 0.3 . Note the extremely high negative correlations - not shown in the table

284 - between components within the BAS approach (it is -0.873 for $\operatorname{Repl}_{\mathrm{BJ}}$ and $\mathrm{Nes}_{\mathrm{BJ}}$ ),

285 and the POD framework as well (-0.864 between $\mathrm{Rich}_{\mathrm{J}}$ and $\left.\mathrm{Repl}_{\mathrm{J}}\right)$. These are

286 empirical indications of interdependence between these components. These results

287 represent only one case study with four variables, and more actual cases should be

288 examined before any far-reaching conclusions can be made about the relationship

289 between diversity components and the environment.

290 7. Monotonicity 
291 Baselga and Leprieur (2015) examined four different scenarios in which an initial

292 configuration was gradually and systematically modified to see if beta diversity

293 components respond monotonically to these changes. The results were inconclusive:

294 components of the BAS and POD differed in performance. We agree with Baselga

295 and Leprieur (2015) that gradual changes of data pattern need not be reflected by

296 monotonic responses under all circumstances. There is one comment only:

297 monotonicity should be distinguished from strict monotonicity, so the POD

298 components were monotonous, while not always strictly monotonic, over the spatial

299 sequence in cases of the extinction and homogenization scenarios.

300 8. Advantages of the POD approach

301 Unfortunately, while focusing on independence and monotonicity issues Baselga and 302 Leprieur (2015) are silent about additional and important features of the POD

303 framework. In this, decomposition of Jaccard dissimilarity is only part of a more

304 general analytical toolkit: $\mathrm{S}_{\mathrm{J}}$ and the two components of $\mathrm{D}_{\mathrm{J}}$ are not restricted to

305 express only a single contrast, the one between similarity and beta diversity along a

306 1D simplex. There are other two possibilities for combining $\mathrm{S}_{\mathrm{J}}$ with $\mathrm{Repl}_{\mathrm{J}}$ or $\mathrm{Rich}_{\mathrm{J}}$.

307 The sum of the first two terms gives the relative richness agreement function:

308

309

$\operatorname{Agr}_{\mathrm{J}}=\mathrm{S}_{\mathrm{J}}+\operatorname{Repl}_{\mathrm{J}}=\frac{a}{a+b+c}+\frac{2 \min (b, c)}{a+b+c}$

311 which represents a contrast with Rich along another 1D simplex. Perhaps more

312 important is the other combination

313

$314 \quad \mathrm{NeS}_{\mathrm{J}}=\mathrm{S}_{\mathrm{J}}+\operatorname{Rich}_{\mathrm{J}}=\frac{a}{a+b+c}+\frac{|b-c|}{a+b+c}$ if $a>0$, otherwise $\mathrm{NeS}_{\mathrm{J}}=0$ 
316 called the relativized nestedness index (Podani and Schmera 2011, 2012, Schmera and

317 Podani 2011). Baselga and Leprieur (2015, p. 1076 right column, top) refer to the left

318 part (i.e. without the $a>0$ condition) as the "similarity index" counterpart of $\beta_{-3}$. The

319 problem is not merely that $\beta_{-3}$ differs from the Repl function - as already said.

320 Equation 6 is not similarity in the conventional sense: it is a sum of similarity $\left(\mathrm{S}_{\mathrm{J}}\right)$ and

321 a fraction of dissimilarity $\left(\right.$ Rich $\left._{\mathrm{J}}\right)$, both contributing with equal weight to pairwise

322 nestedness. Our nestedness concept is clear from this equation: it is determined by the

323 overlap between the species lists of the two sites and by how deeply the list of the

324 poorer site is "embedded" into the species list of the richer site, provided that they

325 have at least one species in common (Podani and Schmera 2012). In other words,

326 nestedness is neither similarity nor dissimilarity, and there is a condition (positive $a$ )

327 involved in its calculation. The contrast between replacement and nestedness is thus

328 expressed on another 1D simplex, clearly showing that - in addition to the

329 disappearance of shared species - species replacement is the only pattern antagonistic

330 to nestedness.

331 Now we can examine the behaviour of the four artificial scenarios examined by

332 Baselga and Leprieur (2015) through our entire simplex approach. The ternary plots

333 of Figure 1 utilize the same information as Figure 4 of the above authors, yet these are

334 in many respects more illuminating demonstrations of the underlying changes of

335 pattern than the graphs of Baselga and Leprieur (2015). The "replacement and loss"

336 series starts with complete self similarity (right corner, Fig. 1a) and losses and

337 replacements direct the process into maximum beta diversity with richness difference 338 dominating over species replacement (i.e., the point representing the last step lies on 339 the beta diversity side, closer to the richness difference corner). The "extinction" 
scenario involves a relatively short trajectory within the plot: the low initial similarity

341 further decreases down to zero while richness difference increases (Fig. 1b). This

342 sequence also ends up with maximum beta diversity, with richness difference even

343 more dominating than in the previous example. The trajectory is near and parallel to

344 the nestedness side, reflecting that species replacement is low and constant all the

345 way. The "homogenization" scenario starts from a position mostly determined by high

346 species replacement (Fig. 1c). The series involves a steady increase in the number of

347 shared species, and the continuous disappearance of unique species. At the end, the

348 species set of one site becomes completely nested within the other: the point

349 representing this site pair falls onto the nestedness side of the triangle. It is closer to

350 the similarity corner, because the number of shared species (i.e. overlap) is higher

351 than the number of species exclusively occurring in the richer site. The "colonization

352 and extinction" scenario begins with a species rich and an empty site, therefore the

353 first point falls right onto the richness difference corner (Fig. 1d). During the

354 colonization process species migrate to the empty site, half of them eventually

355 disappearing from the first one. This involves increasing similarity and species

356 replacement, so that the two sites converge in species richness. In step 17, they are

357 very close to the richness agreement side of the plot (for a total of 99, rather than 100

358 species full coincidence would have been possible). After this point, however, the

359 initially rich site suffers further losses by which it becomes increasingly nested within

360 the "daughter" site. The series finishes at the nestedness side of the plot, with

361 similarity and richness difference equally contributing to the perfect nestedness of the 362 last pair. 


\section{a: Replacement and loss}

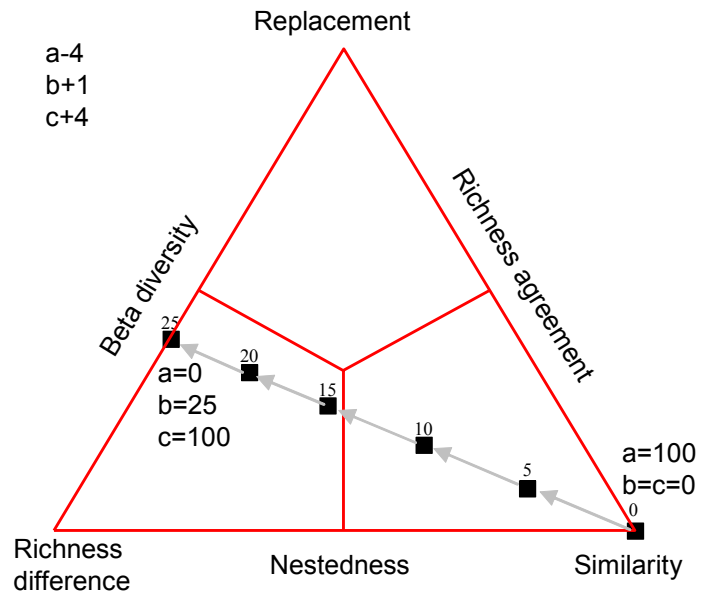

c: Homogenization

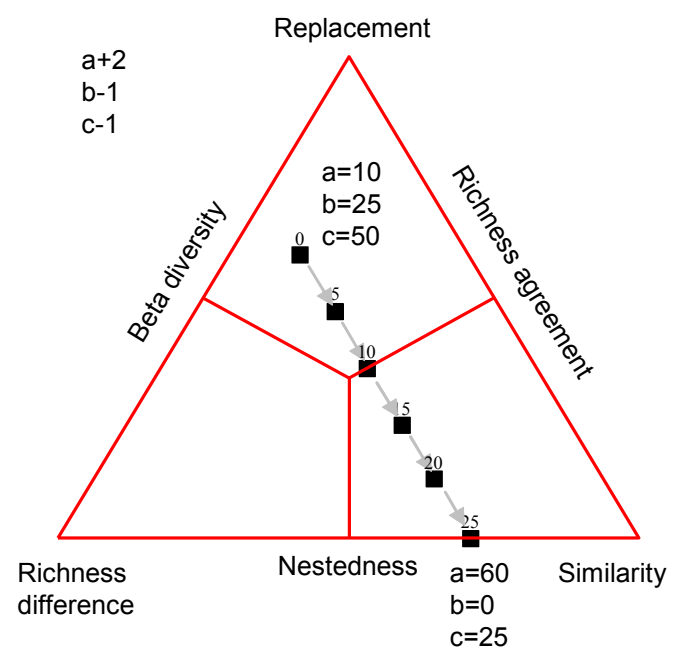

b: Extinction

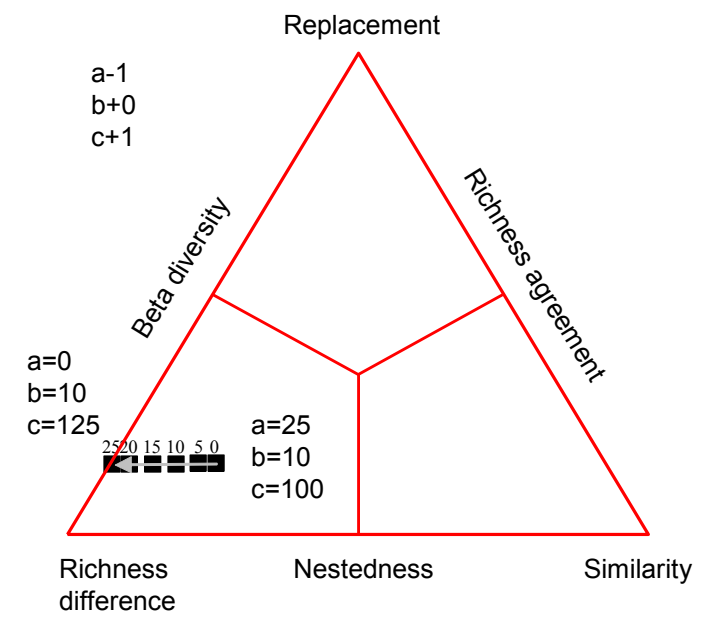

d: Colonization and extinction

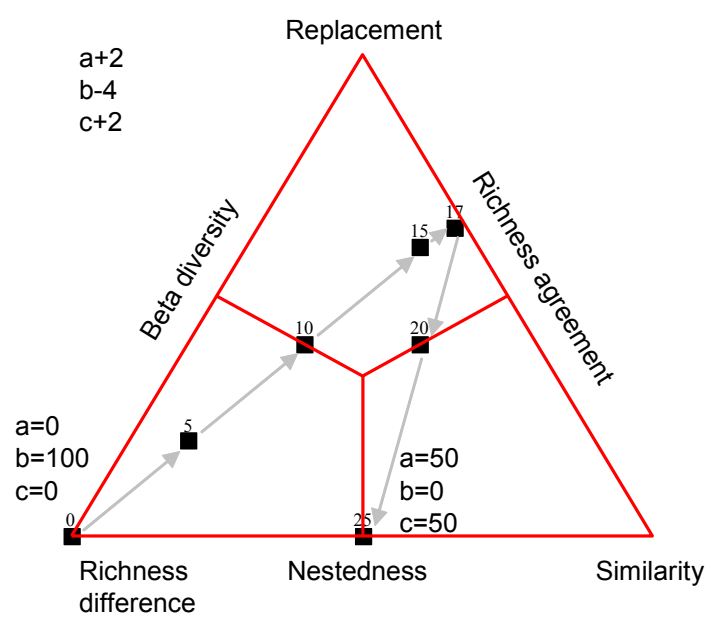

365 Fig. 1. Simplex diagrams illustrating the four 25-step artificial scenarios examined by

366 Baselga and Leprieur (2015). Points labeled by 0 are the initial situations, and then

367 every fifth stage is shown, except for $\mathbf{d}$ to which an extreme position is also added to

368 show that the trajectory does not reach the richness agreement edge. Arrows illustrate 369 direction of the processes. Changes involved in each step are shown in top left of each 370 diagram, the values of $a, b$ and $c$ are shown for steps 0 and 25 only. 
371 For comparison, we also calculated the 2D simplex for the North American fish data

372 as well. Matrix fill in the 171 sites by 549 species data matrix was very low (7.2\%)

373 which is by itself an early indication of high beta diversity. The ternary plot (Fig. 2)

374 confirms this expectation: most of the site pairs are concentrated near the beta

375 diversity edge. Percentage beta diversity is as high as $92.8 \%$, with species

376 replacement contributing with $32.1 \%$ and richness difference with $60.7 \%$. Overall

377 similarity is only $7.2 \%$. Therefore, the centroid of the point cloud falls close to the left

378 edge, nearer to the richness difference than the replacement corner. The anti-

379 nestedness fraction, i.e., the contribution of points which lie exactly on the beta

380 diversity (left) edge is $40.7 \%$ showing that a very large number of site pairs have no

381 species in common at all. We feel that these results are important for a deeper

382 understanding of pattern in the North American fish data: while the BAS approach

383 deliberately ignores richness differences, the POD framework clearly shows that

384 richness difference is the major factor governing beta diversity. 


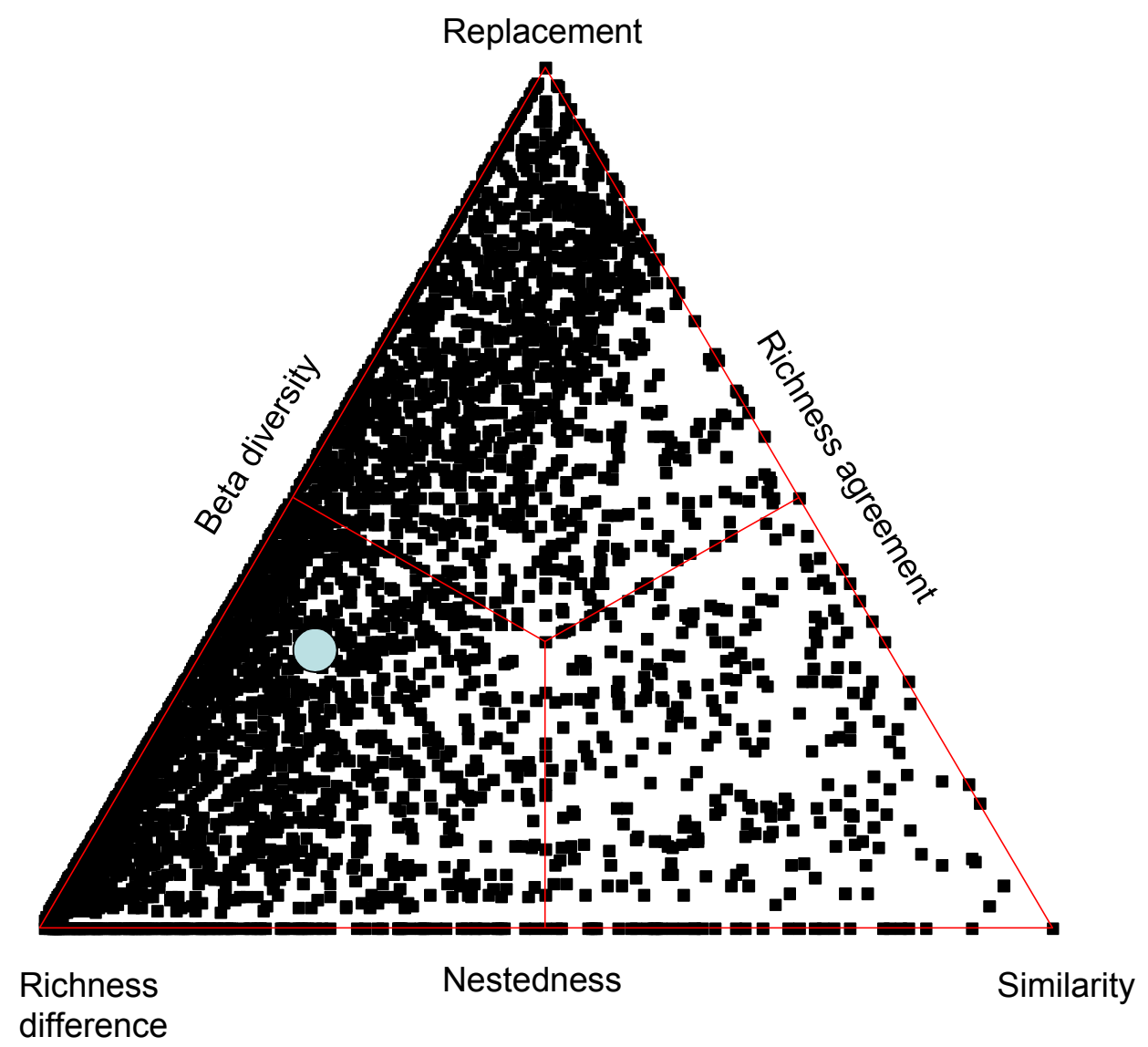

386 Fig. 2. Simplex diagram for the North American fish data. A circle indicates the 387 centroid of the point cloud.

389 Acknowledgements

390 This research was supported by the Hungarian Scientific Research Fund (OTKA 391 104279). 


\section{References}

Almeida-Neto, M., Frensel, D.M.B. \& Ulrich, W. (2012) Rethinking the relationship between nestedness and beta diversity: a comment on Baselga (2010). Global Ecology and Biogeography, 21, 727-777.

Baselga, A. (2010) Partitioning the turnover and nestedness components of beta diversity. Global Ecology and Biogeography, 19, 134-143.

Baselga, A. (2012) The relationship between species replacement, dissimilarity derived from nestedness, and nestedness. Global Ecology and Biogeography, 21, 1223-1232.

Baselga, A. \& Leprieur, F. (2015) Comparing methods to separate components of beta diversity. Methods in Ecology and Evolution, 6, 1069-1079.

Cardoso, P., Borges, P. A. V. and J. A. Veech. (2009). Testing the performance of beta diversity measures based on incidence data: the robustness to undersampling. Diversity and Distributions, 15, 1081-1090.

Cardoso, P., Rigal, F., Carvalho, J.C., Fortelius, M., Borges, P.A.V., Podani, J. and Schmera, D. (2014) Partitioning taxon, phylogenetic and functional beta diversity into replacement and richness difference components. Journal of Biogeography, 41, 749-761.

Carvalho, J.P., Cardoso, P. \& Gomes, P. (2012) Determining the relative roles of species replacement and species richness differences in generating betadiversity patterns. Global Ecology and Biogeography, 21, 760-771.

Carvalho, J.C., Cardoso, P., Borges, P.A.V., Schmera, D. \& Podani, J. (2013) Measuring fractions of beta diversity and their relationships to nestedness: a theoretical and empirical comparison of novel approaches. Oikos, 122, 825-834. 
419 Chao, A., Chiu, C.H. and Hsieh, T.C. (2012) Proposing a resolution to debates on diversity partitioning. Ecology, 93, 2037-51.

421 Chen, Y. 2015. Independence of total species richness and richness difference are not the same thing. Ecological Informatics, 30, 119-123.

Gaston, K.J. \& Blackburn, T.M. (2000) Pattern and Process in Macroecology. Blackwell,, Oxford.

Koleff, P., Gaston, K.J. \& Lennon, J.J. (2003) Measuring beta diversity for presenceabsence data. Journal of Animal Ecology, 72, 367-382.

Legendre, P. (2014) Interpreting the replacement and richness difference components of beta diversity. Global Ecology and Biogeography, 23, 1324-1334.

Podani, J. (2000) Introduction to the Exploration of Multivariate Biological Data. Backhuys, Leiden.

431 Podani, J. (2005) Multivariate exploratory analysis of ordinal data in ecology: pitfalls, problems and solutions. Journal of Vegetation Science, 15, 497-510.

Podani, J. \& Schmera, D. (2011) A new conceptual and methodological framework for exploring and explaining pattern in presence-absence data. Oikos, 120 , $1625-1638$. measures. Ecography, 35, 889-900.

Ricotta, C. and Feoli, E. (2013). Does ordinal cover estimation offer reliable quality data structures in vegetation ecological studies? Folia Geobotanica, 48, 437-

441 Schmera, D. \& Podani, J. (2011) Comments on separating components of beta diversity. Community Ecology, 12, 153-160. 
443 Ulrich, W., Almeida-Neto, M. \& Gotelli, N.J. (2008) A consumer's guide to $444 \quad$ nestedness analysis. Oikos, 118, 3-17.

445 Williams, P.H. (1996) Mapping variations in the strength and breadth of 446 biogeographic transition zones using species turnover. Proceedings of the Royal $447 \quad$ Society London, B 263, 579-588.

448

449 


\section{Appendix 1 - Independence between components of beta diversity}

451 We shall set $n=a+b+c$ as in the main text, while - without loss of generality - we

452 assume here that $b \leq c$. Except the first case, all functions come from the Jaccard

453 family of indices; proofs for the Sorensen family follow a similar logic. Here we

454 adopt the fixed parameter approach (Chao et al. 2012, p. 2040) which checks whether

455 one has information about the value of one component provided that the other

456 component is known. Results of the random parameter approach (Chao et al. 2012, p.

457 2040) and illustrations based on actual data are given in the Supplementary Material.

458 Raw richness difference and raw species replacement

459 These two components of Weiher-Boylen beta diversity are not independent. Since $c$

460 is the sum of $b$ and raw richness difference $\left(D_{r}=c-b\right)$, we have

$461 \quad D_{r}=n-a-2 b$,

462 so that for fixed values of $n$ and $a$, richness difference and replacement mutually

463 constrain each other. To put it differently: raw richness difference can only increase

464 on account of raw species replacement.

465

$B A S$ replacement function and raw richness difference

467 The function $\operatorname{Repl}_{\mathrm{BJ}}=b /(a+b)$ is obviously independent of raw richness difference.

468 For any value of $a$ and $b$, the same result is obtained no matter what values $n$ and $c$,

469 and therefore $D_{r}$ take, since these parameters do not appear in the formula.

POD replacement functions and raw richness difference

472 The function $\mathrm{Repl}_{\mathrm{J}}=2 b / n$ is not independent of raw richness difference. After

473 rewriting we have $\operatorname{Repl}_{\mathrm{J}}=2 b /\left(a+2 b+D_{r}\right)$ so that for a given value $\mathrm{Repl}_{\mathrm{J}}$ and $a, 2 b$

474 and $D_{r}$ mutually constrain each other. If replacement is expressed in relation to the 
richer site, i.e., by Repl $\mathrm{PJ}_{\mathrm{P}}=2 b /(a+c)=2 b /\left(a+b+D_{r}\right)$ (see formula A2 in Appendix 2), it is easy to see by the same reasoning that dependence holds true. In fact, for fixed $n$ and $a$, the remaining $b$ and $c$ mutually constrain each other in any expression incorporating these parameters.

\section{Appendix 2 - Generalized replacement functions}

481 For the Jaccard index family, the new formula for calculating pairwise turnover with respect to the richer site is given by

$$
\operatorname{Repl}_{\mathrm{PJ}}=\frac{2 \min (b, c)}{a+2 \max (b, c)}
$$

This is an extreme case (with $\alpha=0$ ) of the following general formula in which the minimum and the maximum of $b$ and $c$ are weighted by a scale factor $\alpha$ with a range of $[0,1]$ :

$$
\operatorname{Repl}_{\alpha J}=\frac{2 \min (b, c)}{a+2[\alpha \min (b, c)+(1-\alpha) \max (b, c)]}
$$

For $\alpha=1$, we have Baselga's Repl $1_{B J}$ coefficient. For $\alpha=0.5$, equation A2 reduces to the relativized replacement index Repl $_{\mathrm{J}}$. Thus, it may be conceived as a function intermediate between two extremes which consider the number of unique species asymmetrically. In order words, Repl $\mathrm{J}_{\mathrm{J}}$ measures turnover with respect to the total species richness of the two sites. If $\alpha<1$, then both $b$ and $c$ are considered, and therefore the measure is not independent of raw richness difference (see Appendix 1

498 for proofs, and Supplementary Material for graphical demonstrations). 
499 For the Sorensen family of indices we may also define a general formula with $\alpha$ 500 ranging from 0 to 1 :

$$
\operatorname{Repl}_{\alpha \mathrm{S}}=\frac{\min (\mathrm{b}, \mathrm{c})}{\mathrm{a}+\alpha \min (\mathrm{b}, \mathrm{c})+(1-\alpha) \max (\mathrm{b}, \mathrm{c})}
$$

503

504 If $\alpha=0$, we get the analogue of equation A1, known as the Savage index (its

505 complement is called the Braun-Blanquet similarity, see e.g. Háyek 1994, Tulloss

506 1997). For $\alpha=1$, it is easy to see that the general formula becomes identical to the

507 Simpson dissimilarity function, while $\alpha=0.5$ yields the replacement component of

508 Sorensen dissimilarity in the POD framework

509

$510 \quad \operatorname{Repl}_{\mathrm{S}}=\frac{\min (\mathrm{b}, \mathrm{c})}{\mathrm{a}+0.5 \mathrm{~b}+0.5 \mathrm{c}}=\frac{2 \min (\mathrm{b}, \mathrm{c})}{2 \mathrm{a}+\mathrm{b}+\mathrm{c}}$

512 not discussed in this paper. This latter index is therefore a replacement measure with

513 respect to the total number of presences in the two sites.

514 Note that general formulae similar to A2 and A3 have been known from numerical

515 ecology. For example, we can mention the intermediate coefficient suggested by Faith

516 (1984) as generalized by Podani (2000, p. 108). This represents a continuum from

517 Kendall dissimilarity to the Manhattan metric.

519 References to appendices

520 Chao, A., Chiu, C.H. and Hsieh, T.C. (2012) Proposing a resolution to debates on 521 diversity partitioning. Ecology, 93, 2037-51. 
522 Faith, D. P. (1984) Patterns of sensitivity of association measures in numerical taxonomy. Mathematical Biosciences, 69, 199-207.

524 Hayek, L.-A. C. (1994) Analysis of amphibian biodiversity data. Measuring and

525 Monitoring Biological Diversity. Standard Methods for Amphibians. (eds. W. R.

526 Heyer et al.), pp. 207-269. Smithsonian Institution, Washington, D. C.

527 Podani, J. 2000. Introduction to the Exploration of Multivariate Biological Data.

$528 \quad$ Backhuys, Leiden.

529 Tulloss, R.E. (1997) Assessment of similarity indices for undesirable properties and a 530 new tripartite similarity index based on cost functions. Mycology in Sustainable 531 Development: Expanding Concepts, Vanishing Borders. (eds. M.E. Palm \& I.H. $532 \quad$ Chapela), pp. 122-143. Parkway Publ., Boone, NC.

533

534

535 